\title{
Frontières
}

\section{Les services spécialisés en santé mentale pour des personnes âgées du CSSS Cavendish. Déjà 10 ans}

\section{Kareen Nour, Véronique Billette et Alan Regenstreif}

Volume 25, numéro 1, automne 2012

Le vieillissement et sa diversité

URI : https://id.erudit.org/iderudit/1018235ar

DOI : https://doi.org/10.7202/1018235ar

Aller au sommaire du numéro

\section{Éditeur(s)}

Université du Québec à Montréal

ISSN

1916-0976 (numérique)

Découvrir la revue

Citer cet article

Nour, K., Billette, V. \& Regenstreif, A. (2012). Les services spécialisés en santé mentale pour des personnes âgées du CSSS Cavendish. Déjà 10 ans. Frontières, 25(1), 152-178. https://doi.org/10.7202/1018235ar
Résumé de l'article

Plusieurs CSSS du Québec réfléchissent sur l'organisation des soins et services en santé mentale pour la clientèle âgée afin de mieux les desservir. Certains ont souligné leur intérêt à implanter un modèle organisationnel de services en santé mentale qui s'apparente à celui du CSSS Cavendish-CAU. Ce modèle, en place depuis plus d'une dizaine d'années, fut développé grâce à l'expertises cliniques et aux meilleures pratiques dans le domaine. Avant d'exporter ce modèle novateur de services, il apparaissait important d'analyser son évolution à travers le temps. Une analyse détaillée de plusieurs sources de données (ex. documents de travail, entrevues individuelles, focus groups, etc.) a permis de constater que les services imaginés en 1999 ont passablement changés. Cette analyse souligne plusieurs transformations positives telles que l'amélioration du continuum de soins, l'augmentation et la diversification du panier de services, mais également des changements de paradigmes et de pratiques sont questionnables. Le Plan d'action (2005) du MSSS a évacué deux concepts fondamentaux des services spécialisés en santé mentale 60 ans et plus (SSSM60+), soit l'autonomie psychosociale et les comportements à risques qui sont à la base d'une intervention plus sociale que médicale. Une attention particulière doit être accordée à ces concepts afin de les remettre à l'avant plan dans l'offre de services. 


\section{LES SERVICES SPÉCIALISÉS EN SANTÉ MENTALE POUR DES PERSONNES ÂGÉES DU CSSS CAVENDISH. DÉJÀ 10 ANS.}

Kareen Nour, Ph.D.

Agente de planification, de programmation et de recherche, Direction de santé publique de la Montérégie; Chercheure associée au Centre de recherche et d'expertise en gérontologie sociale (CREGES), CSSS Cavendish - CAU

Véronique Billette, Ph.D. Coordonnatrice de l'équipe Vieillissements, exclusions sociales et solidarités (VIES); Centre de recherche et d'expertise en gérontologie sociale (CREGÉS), CSSS Cavendish - CAU

Alan Regenstreif, DIA, MSW, TSP Coordinateur, Pratique de pointe en santé mentale et vieillissement, CSSS Cavendish - CAU

\section{RÉSUMÉ}

Plusieurs CSSS du Québec réfléchissent sur l'organisation des soins et services en santé mentale pour la clientèle âgée afin de mieux les desservir. Certains ont souligné leur intérêt à implanter un modèle organisationnel de services en santé mentale qui s'apparente à celui du CSSS Cavendish-CAU. Ce modèle, en place depuis plus d'une dizaine d'années, fut développé grâce à l'expertises cliniques et aux meilleures pratiques dans le domaine. Avant d'exporter ce modèle novateur de services, il apparaissait important d'analyser son évolution à travers le temps. Une analyse détaillée de plusieurs sources de données (ex. documents de travail, entrevues individuelles, focus groups, etc.) a permis de constater que 
les services imaginés en 1999 ont passablement changés. Cette analyse souligne plusieurs transformations positives telles que I'amélioration du continuum de soins, I'augmentation et la diversification du panier de services, mais également des changements de paradigmes et de pratiques sont questionnables. Le Plan d'action (2005) du MSSS a évacué deux concepts fondamentaux des services spécialisés en santé mentale 60 ans et plus (SSSM60+), soit l'autonomie psychosociale et les comportements à risques qui sont à la base d'une intervention plus sociale que médicale. Une attention particulière doit être accordée à ces concepts afin de les remettre à l'avant plan dans l'offre de services.

ABSTRACT

Several CSSS in Quebec are reflecting on the organization of mental health care and services for older clients in order to better serve this clientele. There has been an interest in implementing the organizational model of mental health services similar to the one at the CSSS Cavendish-CAU. In existence for over a decade, this model was developed with the expertise and clinical best practices in the field. Before introducing this model of innovative services elsewhere, it seemed important to analyze its evolution over time. A detailed analysis of data from several sources (e.g. working papers, individual interviews, focus groups, etc.) found that the services, as planned in 1999, have changed somewhat. This analysis highlights several positive changes such as the improvement of the continuum of care, an increase and diversification of the range of services, but likewise other philosophical changes are questionable. The Plan d'action (2005) of the MSSS has disposed of two fundamental concepts of the specialized mental health services for adults 60 years and more (SSSM60+), that of psychosocial autonomy and risk behaviours, that are the basis for an intervention which is more social than medical. Particular attention should be paid to these concepts in order to return them to the forefront in the provision of services. 
MOTS CLÉS: Vieillissement - santé mentale - meilleurs pratiques évaluation

KEYWORDS: Aging - mental health - best practices - evaluation

C'est connu, la population du Québec vieillit (Ministère de la Famille et des Aînés, 2008). Ce changement démographique va en s'accentuant et on estime que le tiers des personnes âgées de demain présenteront des problèmes de santé mentale $(\mathrm{PSM})^{1}$ qui nécessiteront des services spécialisés dans les prochaines décennies. À l'heure actuelle, c'est plus d'un aîné sur quatre qui présente un PSM (Bartels et al., 2004). Pour le réseau de la santé et des services sociaux, offrir des services adéquats à cette clientèle représente un défi colossal, car elle est, bien plus souvent qu'autrement, sous desservie ou sous diagnostiquée (Cohen, 2001). Au Canada, depuis près de 10 ans, on préconise une approche biopsychosociale à domicile centrée autour des besoins globaux des aînés aux prises avec des PSM vivant dans la communauté et ce, offerte par une équipe multidisciplinaire spécialisée (Kirby \& Keon, 2004; Romanow, 2002). Au Québec, comme dans les autres provinces canadiennes, ce type d'approches destinées spécifiquement aux aînés demeure rare.

À notre connaissance, seul le Centre de santé et de services sociaux (CSSS) Cavendish-Centre affilié universitaire (CAU) offre, depuis plus de 10 ans (1999), des services spécifiques en santé mentale pour la clientèle âgée de 60 ans et plus de son territoire (SSSM60+) (Nour et al., 2011). En 1998, lors d'une évaluation des besoins réalisée sur le territoire du CSSS, il apparaissait clairement que les personnes âgées vivant avec des problèmes de santé mentale n'étaient pas suivis par les services à domicile, ni par d'autres départements du CSSS. C'est suite à ce constat que les SSSM60+ sont nés. Lorsqu'ils ont été développés en 1999-2001, ces services se voulaient à la fine pointe, c'est-à-dire qu'ils étaient basés sur les expertises cliniques ainsi que sur les meilleures pratiques soulignées par les écrits scientifiques et des documents officiels nationaux et internationaux dans le domaine (Nour et al., 2010). Leurs objectifs des SSSM60+ étaient de trouver des solutions adaptées aux besoins multiples des usagers, de prévenir la détérioration de leur situation, de préserver leur indépendance et d'identifier les facteurs environnementaux qui peuvent influencer leur 
santé mentale. Le service de pointe voulait donner une plus grande place aux aspects psycho-sociaux basée sur leur évaluation psychosociale. Cela allait à contre-courant d'une vision médicalisé de la santé mentale qui impliquait la nécessité d'un diagnostic médical pour déterminer les besoins psycho-sociaux. Les services développés étaient novateurs, basés sur les expériences d'une équipe d'intervenants dédiés aux problèmes de santé mentale chez une clientèle âgée. Le développement d'outils permettant l'évaluation des besoins à partir de comportements à risque et la restructuration des services de soutien à l'autonomie même en l'absence de problème de santé physique a permis une toute autre offre de services adaptée à la population visée. Ils s'adressaient à la clientèle âgée, présentant des comportements à risque et vivant autant avec un problème de santé mentale chronique qu'un problème transitoire.

Actuellement au Québec, plusieurs CSSS réfléchissent sur le type et les modalités de prestation de services en santé mentale pour cette clientèle âgée. Certains d'entre eux, soutenu par l'Agence de la santé et des services sociaux (ASSS) de Montréal, ont souligné leur intérêt à implanter au sein de leur établissement un modèle organisationnel de services en santé mentale qui s'apparente de près au modèle actuel (2010) du CSSS Cavendish-CAU (ASSS, 2011). Toutefois, avant d'exporter ce modèle de services, il apparaissait important d'analyser l'évolution de ces services à travers le temps. En effet, entre l'époque de sa création (1999) et aujourd'hui (2010), plusieurs modifications majeures ont eu lieu dans le réseau de la santé et des services sociaux. Que sait-on des transformations qui se sont opérées sur ces services au cours des années? Comment ont-elles affectés l'accessibilité (ex. rejoindre la population cible), la disponibilité (ex. quantité de services et ressources) et la qualité (ex. modalités de prestation) des services offerts?

Parmi les transformations majeures, on ne peut passer sous le silence, la réorganisation, amorcée en 2004 avec la loi 25 (Loi sur les agences de développement de réseaux locaux de services de santé et de services sociaux), des établissements de santé et de services sociaux qui ont été associés par territoires pour créer des CSSS responsables du bien-être et de la santé de la population desservie. Par ailleurs, en 2005, le ministère de la Santé et des Services sociaux (MSSS) présentait son Plan d'action en santé mentale 2005-2010 qui a entraîné une 
refonte majeure de l'organisation et de la prestation des services en santé mentale pour tous les âges. Au niveau du CSSS Cavendish, ce plan a amené l'intégration des services spécifiques de santé mentale offerts aux aînés (SSSM60+) au sein du Programme de santé mentale adulte (PSMA) afin d'assurer un meilleur continuum de services.

Certes ces réformes visent une optimalisation des services offerts aux personnes vivant avec des problèmes de santé mentale, notamment les aînés. Toutefois, il ne faut pas perdre de vue que ces transformations des services publiques prennent aussi place dans des conjonctures sociopolitique et économique particulières (mondialisation des instances de gouvernance, amincissement du champ d'action des États-Nations, récessions, déficits budgétaires à combler, réduction des services à la population, etc.) qui entraînent des répercussions sur l'ensemble des populations mais particulièrement sur les personnes âgées et les services qu'elles reçoivent (Phillipson, 2012; Jenson, 2004; Bickel et Cavalli, 2002). Ce contexte entraîne une recherche d'efficacité rentable, une recrudescence des discours associés aux impératifs économiques pour déterminer l'ampleur et la nature des soins qui seront offerts à la population.

Alors, après tant de transformations, que reste-t-il de ses services spécialisés imaginés et implantés en 1999? C'est la question qui a intéressé une équipe de chercheurs universitaires, d'intervenants et de gestionnaires du CSSS Cavendish-CAU. Est-ce que les services offerts actuellement sont effectivement ceux imaginés et conçus en 1999? Sont-ils toujours novateurs, et sont-ils exportables? Est-ce que les changements de conjoncture des dernières années les ont améliorés? Rejoignent-ils toujours leurs objectifs?

\section{OBJECTIFS DU PROJET}

Cet article présente une analyse de l'implantation des services spécialisés offerts aux aînés vivant avec un PSM sur le territoire du CSSS Cavendish-CAU. Elle vise à répondre à diverses questions afin de bien s'assurer que les services offerts rejoignent la philosophie, les valeurs, les mandats et les objectifs fixés il y a 10 ans (ex. valoriser les enjeux sociaux et psycho plutôt que les médicaux, miser sur le dépistage et l'outreach, de faire reconnaître les difficultés d'autonomie qui ne sont 
pas reliées à la santé physique, d'organiser les services en fonction des besoins du client, etc.) (Dunn \& Bentall, 2007; Bishop \& Vingilis, 2006; Calsyn et al., 2005; Health Education Unit Communication, 2002; Condandriopoulos, et al., 2000).

\section{MÉTHODES DE COLLECTE ET D'ANALYSE DE DONNÉES}

Diverses méthodes de collecte de données ont été utilisées. Premièrement, une analyse documentaire a été réalisée sur la population cible, les services prévus, l'intensité des services, les modalités de prestation ainsi que les ressources connexes et matérielles. Les informations liées aux SSSM60+ et son évolution, ont été recueillies à partir de tous les documents produits (i.e. articles, documents de travail, d'informations et de promotion) à travers les années. Environ une trentaine de documents ont été lus et étudiés.

Deuxièmement, nous avons eu accès à des données provenant des bases d'informations des archives du I-CLSC ${ }^{2}$, soit des statistiques que les intervenants doivent compléter sur chaque intervention reliée aux usagers qu'ils suivent. Ces données couvraient la période de 2004 à 2009. Peu de données étaient disponibles sur la période avant 2004. Des rapports sporadiques ont été réalisés par l'archiviste du CSSS. L'entrée, le nettoyage et l'analyse de quelques données ont été réalisés à l'aide des logiciels EXCEL et SPSS. Des analyses quantitatives descriptives ont été faites. Près d'une cinquantaine de tableaux présentant I'offre de services des SSSM60+ ont été analysés.

Troisièmement, quatre entretiens individuels, d'une durée de 60 minutes, ont été réalisés auprès de deux responsables des SSSM60+ incluant la responsable du développement initial de ces services. Ils ont été menés par la coordonnatrice de recherche à l'aide d'un guide d'entretien contenant une dizaine de questions ouvertes, abordant divers sujets (ex. population cible et rejointe, modalités de services et disponibilités, collaboration entre les programme et la communauté, fusion d'établissements et de programmes). Puis, deux focus groups, animés par la chercheure principale, de 90 minutes ont été réalisés. Une douzaine d'intervenants qui offrent des SSSM60+ y ont participés volontairement. Un guide contenant une dizaine de questions a servi à mener la 
discussion. Il couvrait sensiblement les mêmes sujets que ceux abordés lors des entretiens individuels. Le premier groupe a eu lieu au CLSC Notre-Dame-de-Grace\Montréal Ouest (NDG/MO) et le second, au CLSC René-Cassin, soit les deux CLSC du CSSS Cavendish-CAU. Les entretiens individuels et les groupes ont été enregistrés puis retranscrits. Différentes étapes ont été mises en œuvre pour organiser, reformuler et reconstruire les transcriptions des entretiens et des notes afin d'effectuer une analyse adéquate du matériel (Bardin, 1977; Crabtree \& Miller, 1999). Le matériau qualitatif a été soumis à une analyse qualitative de contenu selon les règles de Thomas (2006 dans Blais \& Martineau, 2007). Le logiciel QDA Miner a été utilisé aux fins d'analyse. Pour l'étude pilote, un échantillon d'usagers $(n=31)$ ont été rencontrés à trois reprises dans l'espace de 6 mois, pour explorer leur évolution dans les SSSM60+ (détail dans Nour et al., 2011).

\section{RÉSULTATS}

Les résultats sont présentés selon les quatre grandes dimensions des SSSM60+ soit les modalités de prestation, la population cible, les services et les ressources. Ils tentent de dépeindre pour chacun des éléments ce qui s'est déroulé au cours de 10 derrières années.

\section{Modalités de prestation}

Dès leur mise en place en 1999, les SSSM60+ souhaitaient développer une approche différente pour mieux répondre aux besoins complets de cette clientèle. Novatrice à l'époque, cette approche proposait une philosophie et un modèle d'intervention différents, ainsi qu'une équipe multidisciplinaire qui offrait des services de santé mentale à domicile.

\section{Philosophie et modèle de prestation de services}

En $1999 .$.

Les SSSM60+ ont élargi la notion de perte d'autonomie physique ou cognitive, barème pour l'obtention de services à domicile, pour y inclure la perte d'autonomie psychosociale soit celle causée par un PSM. Selon 
les responsables des SSSM60+, cette nouvelle perspective psychosociale était devenue essentielle car elle permettait aux aînés aux prises avec un PSM de recevoir des services à domicile qui leur étaient habituellement refusés car ils ne vivaient pas avec une perte d'autonomie physique ou cognitive. Par exemple, les usagers pouvaient recevoir du soutien pour les activités de la vie domestique visant à accroître leur autonomie psychosociale (ex. participer à des activités hors de la maison) ce qui n'était pas possible auparavant. De plus, cette approche a permis de rejoindre une clientèle qui ne voulait pas se présenter au CLSC pour des services de santé mentale. En 2003, Miranda et al. ont précisé que cette stratégie rejoignait le besoin exprimé par une étude nationale portant sur les soins à domicile qui soulignait l'urgence de développer des services de maintien à domicile pour les personnes âgées souffrant de PSM et n'ayant pas de perte d'autonomie physique ou cognitive.

Le concept des comportements à risque a aussi été intégré dans les SSSM60+ dès 1998. Les comportements à risque (négligence de soi, abus de substance, comportements agressifs, détresse émotionnelle, etc.) amènent un dysfonctionnement au niveau personnel et social qui présente un danger pour la santé et la sécurité de l'individu lui-même et celle des autres (Nikolova, et al., 2004). Leurs présences déstabilisent les environnements sociaux et physiques, compromettent l'autonomie et le maintien à domicile. Ces comportements deviendront au cœur du dépistage, de l'intervention et du suivi des usagers qui vivent avec un PSM au CSSS. L'approche poursuivit visait donc la réduction des comportements à risque et non seulement la réduction des symptômes associés au diagnostic de santé mentale. Elle a fait en sorte d'élargir l'accès aux SSSM60+ à des personnes qui n'avaient pas reçu un diagnostic relié à la santé mentale mais qui présentaient des comportements à risque (Nikolova et al., 2004 ; Dallaire et al., 2003). Ainsi, I'objectif de cibler la diminution des comportements à risque visait à soutenir les usagers qui refusaient de divulguer leurs antécédents médicaux ou qui refusaient les aspects médicaux des interventions (ex. traitements pharmacologiques).

\section{En $2010 \ldots$}

La situation avait changé. En fait, le Plan d'action ministériel en santé mentale 2005-2010 a eu un impact important sur l'évaluation des 
demandes de services, sur l'orientation et l'intervention auprès des aînés. Les demandes de services sont dorénavant traitées par le guichet d'accès ${ }^{3}$, un lieu où s'effectuent l'évaluation et l'orientation de nouvelles demandes de services par des intervenants de l'équipe de première ligne, selon les critères d'accès établis par le Plan d'action. Actuellement, le modèle d'intervention prédominant est le Recovery Model, soit de la réadaptation psychosociale qui vise à restaurer, maintenir et améliorer la qualité de vie des personnes avec des PSM en les aidant à maintenir, développer et utiliser des habiletés sociales et fonctionnelles pour vivre, apprendre et travailler dans la communauté avec le plus d'autonomie et de satisfaction possible (Anthony, 1993). Il est aussi interprété comme un modèle de rétablissement où l'élément central demeure l'identification et la réalisation progressive d'un projet personnel, en misant sur les forces de la personne (Repper \& Perkins. 2006). Dès lors, l'approche favorisant le dépistage des comportements à risque est évacuée des critères d'admissibilité et des démarches d'évaluation au profit des outils apparentés au Recovery Model. À I'heure actuelle, cette approche est peu utilisée par les intervenants comme modalité d'intervention et ce, malgré le fait que les comportements à risque sont très présents chez la clientèle (la majorité présente entre 3 et 6 comportement à risque sur un maximum de 10) (Nour et al., 2011). L'outil de dépistage et d'intervention qui avait été développé pour évaluer ces comportements à risque, le psychogeriatric and risk behaviour assessment scale (PARBAS) (Nikolova, et al., 2004) est maintenant optionnel à compléter par les intervenants, ce qui va à I'encontre des recommandations de divers experts (ASSS, 2011). Pour ce qui est de l'autonomie psychosociale, le concept demeure une préoccupation des intervenants qui travaillent avec des aînés aux prises avec un PSM sévère, avec ou sans diagnostic et teinte encore leur pratique, selon eux.

\section{Composition d'équipe}

En $1999 .$.

Les services sont offerts par une équipe multidisciplinaire. Prouvée comme une pratique exemplaire pour répondre aux besoins de la clientèle (AOA, 2001), on retrouvera au cours des premières années, dans l'équipe des SSSM60+, des infirmières, des intervenants sociaux, des auxiliaires et un psychiatre consultant. Les ergothérapeutes ont été 
intégrés peu de temps plus tard. Les données amènent à constater (Nour et al, 2011) qu'il semblait difficile d'avoir des équipes complètes et stables capables d'offrir l'ensemble les services prévus et de répondre aux besoins des usagers.

\section{En $2010 \ldots$}

Bien qu'il y ait eu une augmentation graduelle du nombre et du type d'intervenants (ex. psychologue, bénévoles impliqués) et ce, proportionnellement à la croissance du nombre d'usagers dans les SSSM60+, les intervenants en 2010 mentionnent une insatisfaction face au nombre de ressources. La même réalité qu'en 1999. Des prêts de personnels inter-programmes (ex. ergothérapeute du maintien à domicile) permettent parfois de pallier en partie à l'instabilité de l'équipe.

\section{Lieux d'intervention}

En $1999 . .$.

De par la philosophie d'intervention, $91 \%$ des SSSM60+ étaient offerts à domicile. Les services à domicile étaient un aspect important, une valeur même des services offerts aux aînés vivant avec un PSM et ce, même si elles étaient en mesure de se déplacer au CLSC. Les rencontres face à face avec l'usager étaient donc privilégiées. Offrir des soins à domicile était, et est encore, un indicateur prometteur d'accessibilité et de suivi des clientèles ayant des troubles mentaux (MSSS, 2010; Bernstein \& Hensley, 1993).

\section{En $2010 \ldots$}

Les interventions ont migré du domicile au CLSC en raison de la nouvelle philosophie d'intervention du Recovery Model (2005). En effet, en 2010 , presque tous les usagers ont été rencontrés au CLSC $(95.8 \%)$ et près de la moitié également à domicile (41.7\%). Le mode d'intervention privilégié a également changé: les rencontres face à face avec l'usager, ont cédé tranquillement la place à des interventions téléphoniques de plus en plus fréquentes, sans toutefois les dépasser. Le nombre croissant et la complexité des usagers suivis par chaque intervenant, les a amener à réduire leurs déplacements afin d'avoir plus de temps pour discuter avec chacun de leurs usagers...au téléphone. 


\section{Population ciblée et rejointe}

La seconde question est à savoir si la population ciblée par les services est celle rejointe. L'analyse porte sur les critères d'admissibilité aux SSSM60+ ainsi que sur la population rejointe. Étape incontournable pour obtenir des services, l'évaluation des critères d'admissibilité détermine la population visée par les services. Bien qu'il soit toujours demeuré très clair que les services s'adressent à la population âgée de plus de 60 ans, les autres critères d'admissibilités pour bénéficier de ces services ont passablement changé au cours des années.

\section{Population ciblée}

\section{En $1999 . .$.}

Le critère d'admissibilité central était la présence de comportements à risque observables. Ce critère avait été identifié par les intervenants du milieu qui mentionnaient que beaucoup d'usagers n'avaient pas nécessairement de diagnostic psychiatrique et ce pour diverses raisons, mais avaient de grands besoins de services en lien avec leurs comportements à risque qui représentaient plus leur portrait complet de leur situation (autant physique que social) (AOA, 2001). Il avait été convenu que les usagers ne devaient donc pas obligatoirement avoir reçu un diagnostic psychiatrique ou encore être suivi par un psychiatre pour avoir accès aux services. De plus, l'expérience clinique a mené à exclure des SSSM60+ les personnes atteintes de déficits cognitifs étant donné qu'ils nécessitaient des interventions différentes (Moscovitz, 2000) et étaient déjà suivi dans le département soutien à domicile. Les usagers pouvaient présenter des problématiques en lien avec une ou des situations transitoires (ex: deuil, dépression) ou présenter un problème de santé mentale chronique. Malheureusement, puisque n'étant pas le critère d'admissibilité, les données disponibles ne nous permettent pas de connaitre la répartition des deux catégories d'usagers desservies selon le type de problème de santé mentale vécu (chronique ou transitoire). L'âge était un critère également, ou les usagers admis devaient avoir 60 ans et plus. 
Ces critères ont connu une transformation importante avec l'arrivée du Plan d'action (2005) qui est venu déterminer le principal critère d'accès aux services de santé mentale: un diagnostic ou une impression diagnostique ${ }^{4} d^{\prime} u n$ trouble mental. Les comportements à risque n'étaient plus dans la liste des critères, ce qui représente un changement majeur d'accessibilités aux services. Les usagers pouvaient encore présenter des problématiques en lien avec une ou des situations transitoires (ex: deuil, dépression) ou présenter un trouble mental plus chronique. Aucune donnée administrative n'est facilement disponible sur la répartition des usagers en lien avec ces problématiques (transitoire vs chronique), mais les intervenants mentionnent suivre une clientèle plus lourde. Quelques données semblent leur donner raisons: le pourcentage d'usagers suivis vivant avec de la schizophrénie et des troubles psychotiques a augmenté de $20 \%$ au cours de 10 années. Il est à noter que, par l'intégration des SSSM60+ au PSMA (2008), I'âge a cessé d'être un critère d'admissibilité ce qui a amené une continuité dans les services de santé mentale de l'âge adulte jusqu'à la vieillesse.

\section{Population rejointe}

\section{En 1999...}

Les SSSM60+ rejoignaient la population ciblée au départ. La première étude (Lee Maurel, 1999) fournit un portrait d'un échantillon de la clientèle suivie à cette époque $(n=25)$. Les usagers étaient surtout des femmes $(84 \%)$, veufs ( $48 \%)$, vivant en grande majorité seul $(60 \%)$ et ont un revenu de pension (44\%), parfois accompagné d'économies personnelles (36\%). Ils avaient de la famille dans la région (60\%) mais on ne déteint pas d'information sur la qualité du réseau social. Presque $90 \%$ présentent une polypathologie. Une proportion de $54 \%$ des usagers n'a pas diagnostic «officiel» en santé mentale et 56\% souffrent de dépression. L'ensemble des usagers présentaient plusieurs comportements à risque (dont $30 \%$ ne suivent pas leur prescription et $22 \%$ qui refusent un suivi psychiatrique) et une perte d'autonomie résultant de leur problème psychiatrique ou en raison de leurs pertes physiques et cognitives. Selon les intervenants, les usagers sont perçus comme étant plus difficiles et les cas plus exigeants que les autres 
usagers suivis par le CLSC. Ils se retrouvent souvent en situation de crise ou qui mettent leur vie en danger.

\section{En $2010 \ldots$}

C'est sensiblement le même portrait de la clientèle. Une plus grande proportion d'hommes est suivie par les services (près de $30 \%$ ). Ils ont en moyenne 73 ans, vivent en majorité seul (83\%), présentent des problématiques de négligence de soi ( $52 \%$ ) ou de sécurité financière $(42 \%)$. Les usagers allophones sont plus nombreux qu'autrefois, ceci complexifiant l'intervention dans la mesure où les usagers allophones ne comprennent et/ou ne parlent ni anglais, ni français, que ces langues sont celles des intervenants et que le recours à des interprètes s'avère nécessaire. Finalement, $83 \%$ des usagers ont un diagnostic officiel (devenu un critère obligatoire d'admissibilité) (et les autres $17 \%$ sont en attentes de ce diagnostic). Toutefois, la clientèle est toujours considérée aussi exigeante en raison de la complexité de leurs problématiques qui augmentent les défis de l'intervention. (Les données complètes sur la population rejointe se retrouve dans Nour et al., 2011).

\section{Services offerts}

Parler de services, c'est souvent en termes de quantité, de nature et complémentarité. Qu'est-il advenu de ces services en 10 ans?

\section{Quantité de services}

La quantité de services peut être évaluée par le nombre d'interventions effectuées, le nombre d'usagers suivis par les SSSM60+ et le nombre d'usagers sur la liste d'attente pour des SSSM60+.

En $1999 \ldots$

Les données administratives n'étaient pas accessibles sur cette dimension. Toutefois, en 1999, il était convenu que puisque les interventions étaient à domicile et auprès d'une clientèle vulnérable, une certaine intensité de services (ex. un nombre de rencontres intensives et périodiques) allait être mise sur place, particulièrement en début de suivi. 
Au total, environ 13000 interventions ont été effectuées en 2009, pour 453 usagers soit environ 28.7 interventions \année\ usager. Bien qu'il n'y ait pas de ligne directrice quant au nombre d'interventions à offrir par année aux usagers, on constate que le nombre d'interventions totales est en décroissance depuis les années 2000 (en 2006, 15000 interventions; aucune donnée disponible avant). Cette diminution du nombre total d'intervention s'explique principalement par une diminution du nombre d'usagers suivis par les SSSM60+ de 562 usagers en 2006 à 453 usagers en 2009 (population territoriale stable de 22000 aînés). Toutefois, l'intensité des interventions (i.e. nb interventions \année\ usager) est restée semblable avec le temps (28.7 vs 26.7) avec des ressources professionnelles quasi identiques en terme de nombre et de diversité (Nour et al., 2011)

Selon les intervenants, les CLSC sont devenus les points de services principaux pour les aînés aux prises avec un PSM. La réorganisation de services proposée par le Plan d'action (2005) a amené les intervenants des CLSC à assurer le traitement d'une plus grande proportion d'usagers chroniques et stables (ex. trouble de l'humeur) qui ont besoin de suivi à long terme, ainsi que d'usagers, qui habituellement se dirigeaient vers les hôpitaux, soit ceux avec des troubles de santé mentale sévères et persistants (ex. schizophrénie). Ces nouveaux types de clientèles ont eu comme conséquence d'allonger la durée du suivi de chacun des usagers. En effet, une des particularités des SSSM60+ était, et est encore, une durée du suivi très variable et dépendant grandement des besoins des usagers. Or, selon les intervenants, en raison de la complexité des cas actuels, le suivi doit être prolongé, réduisant ainsi l'accessibilité aux services pour de nouveaux usagers.

Pour ce qui est des personnes sur la liste d'attente pour des SSSM60+, les données administratives ne nous permettaient pas de statuer clairement de l'évolution des temps d'attente, ni du nombre de personnes sur sa liste d'attente. Toutefois, les critères établis par le Plan d'action (2005) (7 jours entre la demande de services et le contact par l'intervenant du triage et 30 jours entre la demande et la prise en charge par un intervenant), nous permettent de les comparer aux temps d'attente actuels. En 2011, il a été constaté que chez un échantillon d'usagers, une moyenne de 90 jours (e.t. 104,47) s'étaient écoulés, entre la 
demande et celle du contact avec l'intervenant désigné (Nour et al., 2011). Durant cette période d'attente, I'usager peut toujours contacter I'intervenant du guichet d'accès si nécessaire. Les usagers ayant reçu leurs services dans les temps prévus étaient ceux dont la situation était jugée comme critique par l'intervenant du guichet d'accès (ex. propos suicidaire, risque potentiel pour eux et autrui). Les autres dépassent de loin ceux les temps prévus dans le Plan d'action (2005).

\section{Nature des services}

En $1999 .$.

La base du panier de services a été établie dès les premiers temps de la mise en place des services en 1999. Les services avaient pour but de trouver des solutions adaptées aux besoins des usagers, de prévenir la détérioration de leur situation, de préserver leur indépendance et d'identifier les facteurs environnementaux qui peuvent influencer leur santé mentale (AOA, 2001). Les services offerts étaient classés en deux grandes catégories: 1) un service de consultation à court terme pour les usagers qui présentent des situations problématiques transitoires (ex.: deuil, dépression) et 2) des services pour les personnes aux prises avec des PSM sévères et persistants. Les services étaient offerts aux usagers et à leurs proches aidants. Les intervenants sociaux offraient, à titre d'exemple, des services de gestion de cas, référence vers d'autres ressources, évaluation du risque, gestion des comportements, intervention psychosociale, soutien aux aidants. Les infirmières faisaient le bilan de santé, la promotion de la santé, l'information et l'enseignement, la psychoéducation, les soins à domicile, la gestion de médicaments et des consultations. Les auxiliaires familiales et sociales offraient des soins et l'hygiène personnelle, de l'aide à la préparation de repas, des informations/enseignement, de la stimulation et un soutien émotionnel. Finalement, le psychiatre faisait principalement de l'évaluation et des consultations.

En $2010 \ldots$

Peu de changements ont été effectués sur le panier de services. Seul des services de réadaptation se sont rajoutés, bonifiant le type de services pouvant être offerts: évaluation et traitement des problèmes 
moteurs et neurologiques, évaluation des capacités/incapacités, évaluation du milieu de vie et de ressources, enseignement d'exercices et de principes de déplacements sécuritaires, etc.

\section{Complémentarité des services}

\section{En $1999 .$.}

La réponse aux besoins des usagers des SSSM60+ a parfois requis I'implication d'intervenants en provenance d'autres programmes (ex. physiothérapie, nutritionniste) à l'intérieur du CSSS. Dans de tel cas, les intervenants et les usagers peuvent compter sur la complémentarité des services entre les programmes. Cette collaboration entre les services d'un même établissement de santé permet une meilleure qualité et continuité des soins (AOA, 2001). Les usagers peuvent aussi être transférés d'un programme vers un autre. Par exemple, quand la composante psychiatrique compromet l'offre de services et la réponse aux besoins des usagers desservis par le Programme perte d'autonomie liée au vieillissement (PPALV), les usagers sont orientés vers le département en santé mentale Les intervenants du PPALV peuvent aussi recevoir le soutien des intervenants des SSSM60+.

En $2010 \ldots$

La collaboration entre les programmes existe toujours. Toutefois, elle est jugée par les intervenants comme étant de plus en plus difficile, toujours en raison du nombre d'usagers et de la complexité de leur situation, et ce au détriment de la quantité de services aux usagers. Les discussions entre SM et PPALV sont majoritairement de nature administratives (ex. accessibilité aux services) et non des consultations de soutien mutuel.

\section{Ressources}

En $1999 . .$.

Plusieurs ressources et collaborations ont été mises en place pour assurer le bon fonctionnement des SSSM60+. Au niveau financier, les SSSM60+ ont émergé, du programme de soins à domicile déjà existant 
en CLSC. Ceci a facilité I'allocation des ressources qui, avaient été transférées des services de soins à domicile vers les SSSM60+. En plus du transfert de ces ressources financières, des ressources humaines ont été transférées d'un programme à l'autre. Dès leur création, les SSSM60+ avaient donc leur propre enveloppe budgétaire, ce qui se distingue des autres CSSS. De plus, les SSSM60+ du CSSS disposaient d'un peu d'argent supplémentaire provenant du MSSS en lien avec sa désignation universitaire, désignation permettant aux intervenants de travailler au développement des pratiques et à la recherche.

En $2010 \ldots$

Ces collaborations perdurent, se solidifient et les enveloppes financières demeurent intactes, ce qui est un grand avantage pour ces services. En fait, au fil des années, plusieurs partenariats se sont développés avec 1) le département de psychogériatrie de l'Hôpital général juif de Montréal; 2) la communauté: commerçants, services publics, etc. (via le Projet P.I.E . Prévention-Intervention-Education); 3) la table de concertation en la santé mentale du centre-ouest: droits et services pour les personnes âgées; 4) plusieurs organisations : ex. : Association québécoise pour la réadaptation psychosociale, Coalition canadienne pour la santé mentale des personnes âgées, Association canadienne pour la santé mentale.

Le même financement est toujours disponible. De plus, au cours des années, les SSSM60+ ont reçu du financement du Conseil québécois de la recherche sociale, de la Fondation pour le Bien-Vieillir, du Prix du fonds d'innovation pour les services aux aînés, de la Fondation RBC, etc.

\section{DISCUSSION}

En 10 ans, plusieurs dimensions des SSSM60+ sont restés stables ou ont été renforcés: le panier de services, l'équipe multidisciplinaire, les ressources et partenariat dans la communauté, la collaboration entre les programmes, la croissance des ressources et le continuum de soins. Toutefois, des changements, pour ne pas dire des reculs, importants ont été observés à l'égard de plusieurs aspects des SSSM60+: abandon du concept d'autonomie psychosociale, abandon de l'approche favorisant le dépistage des comportements à risque, accès plus difficile 
aux services en raison du resserrement des critères d'accès, longs délais d'attente et diminution du nombre d'interventions à domicile. Ces constats soulèvent des inquiétudes sur la place accordée aux dimensions psychosociales dans le système de santé et de services sociaux québécois.

\section{Une équipe multidisciplinaire et de nombreux services}

Depuis la création des SSSM60+, l'idée d'une équipe multidisciplinaire offrant tout un panier de services était centrale pour une offre optimale de services. Les années ont passé et à l'heure actuelle, ces services sont encore plus variés et l'équipe s'est renforcée d'intervenants de diverses professions. Ces changements amènent une offre de services bonifiée plus adaptée aux besoins de sa clientèle. Les équipes multidisciplinaires permettent une intervention plus adaptée aux besoins des usagers. Les interventions ne sont ainsi pas que médicale, mais également sociale, nutritionnelle, etc. (AOA, 2001)

\section{Des ressources communautaires précieuses, des collaborations aidantes intra-établissement et une enveloppe budgétaire protégée}

Le SSSM60+ peut compter sur de nombreuses collaborations qui se sont renforcées à travers les années. Ces dernières permettent de pallier pour des services qui ne seraient pas offerts par les SSSM60+ ou même par le CSSS. Les usagers demeurent les grands gagnants de ces collaborations qui assurent une meilleure continuité de services. La collaboration, formelle ou informelle, est crucial pour connecté les différents acteurs à la communauté et planifier ensemble les décisions à prendre (CAMH, 2009). Elle assure ainsi que les interventions demeurent contextualisées, près des usagers, sans coupures dans le soutien social et médical offert. De plus, de par l'enveloppe budgétaire protégée, les SSSM60+ sont assurés. Leur coté revendicateur et novateur, leur permet aussi d'aller chercher des enveloppes budgétaires supplémentaires assurant leur développement et d'être toujours à la fine pointe des meilleures pratiques. 


\section{Une intégration des services... pour une continuité des services.}

L'intégration des SSSM60+ au sein du PSMA depuis 2009 vise à assurer une meilleure continuité des soins et des services de santé mentale. L'orientation vers un programme se fait en fonction du PSM et non selon l'âge. Ainsi, il n'y a pas de rupture de services par le seul fait que l'usager ait atteint l'âge de 65 ans comme c'est le cas dans plusieurs CSSS, ou les personnes âgées perdent le suivi réalisé par des intervenants experts en santé mentale.. L'approche préventive sur laquelle repose l'intégration des services, et qui semble orienter leur développement, fera peut-être en sorte que les adultes vieilliront en meilleure santé physique et psychosociale. Les plus jeunes usagers bénéficient de l'expertise en santé mentale acquise au cours des années concernant les clientèles âgées. Malgré ces avantages indéniables, on peut toujours se demander quels sont les bénéfices de cette intégration pour la clientèle âgée actuelle. Par ailleurs, les impacts de l'approche préventive doivent aussi être abordés sous I'angle de la quantité des services. Cette approche pourrait avoir des impacts sur le développement de nouveaux services, au profit de la clientèle plus jeune.

\section{Où se retrouvent I'autonomie psychosociale et les comportements à risque?}

On observe donc un recul de la notion d'autonomie psychosociale qui était pourtant fondamentale lors de l'émergence des services de santé mentale aux aînés. Il en est ainsi également pour l'approche favorisant le dépistage des comportements à risque qui, conjuguée avec celle de l'autonomie psychosociale, tentait de remettre en question l'approche médicale prédominante au début des années 2000. La venue de Plan d'action (2005) peut être considérée comme un recul important d'une approche qui voulait être biopsychosociale. La présence d'une maladie souligne la tendance encore bien présente de la médicalisation des PSM, même si le Plan d'action reconnaît les impacts de la maladie sur les différents aspects de la vie (familiale, sociale, etc.). Ce virage quant à la place du regard social est préoccupant pour cette clientèle. Est-ce que ce virage amènera une plus grande médicalisation des usagers, sans préoccupation majeure pour les enjeux d'isolement, d'exclusion, de soutien social? 
Le nouveau modèle de prestation de service, la réadaptation psychosociale (Recovery Model) utilisé a été développé et évalué en fonction de clientèles plus jeunes. Les études sur ce modèle auprès de clientèles âgées sont rarissimes. Il est donc difficile de savoir dans quelle mesure chacune des composantes de ce modèle est adaptée aux clientèles âgées. Malgré les valeurs que sous-tend le modèle, pour lesquelles il peut exister un certain consensus social, la prudence face à l'utilisation de ce modèle auprès des aînés est de mise. Le modèle, à la base, vise à aider les personnes vivant avec un PSM à se réinsérer socialement et à réapprendre à travailler. Ces préoccupations sont loin des réalités vécues par les aînés et certains enjeux sociaux propres au vieillissement et aux PSM particulièrement en lien avec les préjugés, la stigmatisation, l'âgisme risque d'être peu abordées: le fait de considérer comme normal que les aînés soient déprimés ou anxieux, le fait de croire qu'il n'y a pas obligation de les traiter puisqu'ils sont âgés, le fait de les percevoir comme des demandeurs de services plus exigeants que les clientèles plus jeunes (CTSM, 2007). Ces enjeux qui les distinguent font qu'il peut-être risqué d'appliquer textuellement un modèle de prestation pour les jeunes à une population âgée.

\section{- Resserrement des critères d'accès et délais d'attentes questionnables.}

Les critères d'accessibilité pour les SSSM60+ ont connu une transformation importante avec le Plan d'action (2005) où un diagnostic ou une impression diagnostique d'un trouble mental devient nécessaire. Ceci a eu comme répercussion de restreindre l'accès aux personnes n'ayant que des comportements à risque ou qui présentaient une perte d'autonomie psychosociale (sans diagnostic médical) et d'ainsi réduire le nombre d'usagers suivis. La priorisation par la présence de comportement à risque était plus porteuse en termes d'interventions requises pour préserver l'autonomie psychosociale. Toutefois, malgré cet accès plus restreint à ceux ayant un diagnostic, selon l'étude pilote (Nour et al., 2011), pour plusieurs usagers participants, les délais d'attente dépassent largement ceux prévus par le Plan d'action. Diverses explications pourraient permettre de comprendre ce phénomène. Quelques usagers participants ont été difficiles à rejoindre soit au moment du triage ou du traitement en raison de déménagement, 
d'hospitalisation ou d'une hésitation à recevoir les services (nonretour des appels). Par ailleurs, il est possible que la priorisation de nouveaux usagers, dont la situation était jugée plus urgente, ait fait en sorte d'allonger le délai d'attente de plusieurs participants aux situations moins critiques. Dès lors, on peut se demander si les délais prévus par le Plan d'action sont réalistes compte de tenu de la disponibilité des ressources de première ligne ou encore, si les mécanismes actuellement en place au CSSS permettent de rencontrer les délais prévus par le Plan.

\section{Des usagers qui reçoivent surtout des services au CLSC}

Les données administratives ainsi que l'étude pilote démontrent que les usagers reçoivent surtout les services au CLSC (Nour et al., 2011). Le pour et le contre du domicile comme lieu d'intervention se pose. D'une part, les intervenants soulignent que le fait de se déplacer tend à augmenter le sentiment d'empowerment chez les usagers qui le font et les aide à s'impliquer dans leur plan d'intervention. D'autre part, la littérature tend à démontrer que les interventions à domicile seraient plus efficaces pour amener une réduction des symptômes de dépression à court et moyen terme (Klug et al., 2010).

Le nouveau modèle de prestation de services dirige les intervenants vers des interventions en établissement, ce qui selon nous, réduira au cours des années (comme la tendance commence à le démontrer) les interventions de type face à face aux profits de celles téléphoniques. Non seulement le modèle amène ce changement mais également plusieurs contraintes accentuent ce phénomène: pour les intervenants, qui manquent de temps et de ressources pour gérer leurs listes de cas trop remplis, les interventions téléphoniques sont rapides, flexibles et sembles efficaces; pour les gestionnaires, les interventions téléphoniques permettent d'assurer que les intervenants sous leur supervision fassent le maximum avec les ressources qu'ils ont; pour les usagers, les interventions téléphoniques permettent de réduire leur déplacement, ce qui peut être un avantage pour ceux ne désirant pas se rendre au CLSC. Malgré tous ces avantages, il est fort à parier qu'une partie de l'alliance thérapeutique intervenant-usagers risque dès lors, de s'effriter. En effet, le contact de type face à face est crucial pour cette 
alliance, qui selon de nombreux écrits est au cœur de la réussite des interventions sur le plan du suivi et sur le plan social, particulièrement avec des populations plus vulnérables (Calsyn et al., 2005). Ce type de contact permet également de mieux identifier l'évolution des comportements à risque. Il est également essentiel pour bien évaluer le contexte dans lequel la personne évolue et pour intervenir sur plusieurs dimensions sociales liées à leur condition (ex. isolement).

En fait, la comparaison entre les services imaginés en 1999 et ceux proposées par le Plan d'action (2005), semble démontrer une différence concernant la dimension «sociale» du biopsychosocial. Le social semble avoir reculé au détriment du bio et du psycho qui prennent toute la place. La valorisation et la facilitation des liens sociaux, de la participation sociale et citoyenne sont des enjeux pour les aînés en général... encore plus pour ceux qui vivent avec des PSM.

\section{Le modèle actuel... plus près du programme du suivi intensif que d'une approche initiale communautaire.}

Le programme de suivi intensif de la clientèle en santé mentale (PACT en anglais pour Program of Assertive Community Treatment) est un autre modèle de référence fondé sur des données probantes. Il préconise un travail d'une équipe interdisciplinaire pour donner, de façon cohérente et intégrée, des traitements psychiatriques ainsi que des services de réadaptation et de soutien à des personnes qui ont des troubles mentaux graves. Les services de suivi intensif s'adressent à des personnes qui présentent des troubles psychotiques (ex. schizophrénie), qui ne bénéficient pas des services usuels, qui sont souvent réhospitalisées, et qui présentent des troubles concomitants (ex. alcoolisme). Ces usagers éprouvent des difficultés très importantes à fonctionner de façon satisfaisante dans la communauté (Association des hôpitaux du Québec, 2004). Les SSSM60+, tel qu'imaginé en 1999, n'adoptaient pas cette perceptive biomédicale (i.e. admissibilité basée sur la présence d'un diagnostic de troubles mentaux graves, suivi assurée par des psychiatres et infirmières), ne visaient pas les mêmes clientèles (ex. problématiques transitoires, comportements à risque, personnes pouvant demeurer dans la communauté avec du soutien) et 
n'utilisaient pas la même approche (ex. approche de l'autonomie psychosociale). Or, avec les changements qui se sont opérés au cours des 10 années, tout porte à croire que l'approche biomédicale prend le dessus, et que le modèle actuel est davantage lié au programme de suivi intensif. Bien qu'intéressant et porteur, ce modèle demeure adapté à une fraction de la clientèle suivi par les CSSS soit la clientèle lourde, hospitalisée, nécessitant un suivi très serré. Cela semble loin de la réalité de clientèle vivant à domicile avec un problème chronique stable ou un problème transitoire.

\section{CONCLUSION}

Les services spécifiques imaginés en 1999 ont passablement changé avec les années et ce en raison de diverses transformations du système de santé et des normes québécoises pour l'accessibilité et le traitement des personnes vivant avec un PSM. En lien avec les meilleures pratiques reconnues pour les services de santé mentale pour les aînés, cette analyse souligne plusieurs transformations positives telles que I'amélioration du continuum de soins, l'augmentation et la diversification du panier de services ainsi que le renfoncement de l'équipe multidisciplinaire et des ressources, du partenariat et des collaborations. Toutefois, d'autres changements sont questionnables. Le Plan d'action (2005) a eu comme répercussion de mettre de côté deux concepts fondamentaux des SSSM60+, soit l'autonomie psychosociale et les comportements à risques. La contribution importante des aspects plus sociaux de ses deux dimensions dans l'offre de services a été remplacée par une visée plus médicale, qui influence directement la sélection des usagers, la nature des interventions et leurs modalités. Sur le plan «vieillissement» et «social», la pertinence du modèle «Recovery» pour les aînés parait donc questionnable.

Notre recherche nous a permis de constater la persistance et la résistance du modèle médical lorsque vient le temps de penser les services pour les personnes âgées vivant avec des problèmes de santé mentale. Le service de pointe a tenté d'amener les services vers une approche davantage axée sur les forces et le potentiel des personnes, sur le social, se positionnant à contre-courant du modèle médical depuis 1999. Une approche permettant l'évaluation des besoins à partir des 
comportements à risque, plutôt que par l'obligation d'un de diagnostic, permettait d'offrir des services sociaux adaptés aux personnes âgées. Ces services ont été mis à l'épreuve par les restructurations, par le Plan d'action (2005-2010), le tout s'inscrivant dans une conjoncture sociopolitique mondiale de réduction des rôles des états, de compression budgétaire et de réduction des services à la population. Dans un tel contexte, la recherche de plan de services efficaces et rentables semble tendre davantage vers une approche médicale et rend la vie difficile à une approche axée sur une évaluation psycho-sociale approfondie et qui requiert de prendre le temps avec les personnes âgées qui ont des besoins particuliers.

Malgré les difficultés à maintenir les pratiques de pointe développées jusqu'à présent, le modèle de service réalisé par l'équipe de pratique de pointe (maintenant appeler: Santé mentale et vieillissement du CSSS Cavendish-CAU suscite l'intérêt des autres CSSS et d'autres institutions sensibles aux situations vécues par les aînés qui vivent des problèmes de santé mentale. Toujours à contre-courant des orientations et plan d'action gouvernementaux, ces pratiques nous semblent plus que jamais pertinentes et le déploiement de moyens pour les maintenir en place nous semble incontournable afin d'éviter de retomber dans le modèle médical qui est d'ailleurs critiqué par plusieurs chercheurs et organisations (MacCourt, 2008; Dallaire, Miranda et al., 2003).

En ce moment, la recherche de pratiques innovantes est la voie de solution pour les difficultés rencontrées par les institutions de santé et de services sociaux toujours compressées mais devant faire face aux besoins de leur population. Loin d'être contre l'innovation des pratiques, nous pensons toutefois que le maintien de celles-ci devrait être une priorité aussi. Le maintien de bonnes pratiques n'est pas un retour en arrière ou un manque d'innovation et doit être considéré afin d'éviter la recherche frénétique de nouveautés.

Dans un contexte où les discours deviennent de plus en plus teintés des préoccupations économiques des états et des gouvernements, il nous semble essentiel de souligner l'importance de maintenir des espaces de réflexion et de recherche, comme le pratique de pointe Santé mentale et vieillissement. Cela permet de s'assurer de la mise en 
place et du maintien de pratiques dont les préoccupations seront davantage en lien avec les besoins psycho-sociaux d'une clientèle vieillissante vivant avec des problème de santé mentale, sujette aux dures réalités de la stigmatisation et de l'exclusion.

\section{BIBLIOGRAPHIE}

AGENCE DE LA SANTÉ ET DES SERVICES SOCIAUX DE MONTRÉAL (2011). Plan d'action ministériel en santé mentale 2005-2010. Santé mentale et vieillissement. Constats et recommandations concernant l'offre de service. Plan de mise en œuvre à Montréal. Phase III. Service de la planification, du développement stratégique et de l'évaluation.

ADMINISTRATION ON AGING (2001). Mental health and elderlies: issues and opportunities, Rockville, États-Unis. Department of Health and Human Services.

ANTHONY, W. A. (1993). «Recovery from mental illness: The guiding vision of the mental health service system in the 1990s», Psychosocial Rehabilitation Journal, vol. 16, n 4, p. 11-23.

BARDIN, L. (1977). L'analyse de contenu, Paris, Presses Universitaires de France.

BARTELS, S. J., B. FORESTER, K. T. MUESER, K. M. MILES, K. R. DUMS, S. I. PRATT, A. SENGUPTA, C. LITTLEFIELD, S. O'HURLEY, P. WHITE et L. PERKINS (2004). «Enhanced skills training and health care management for older persons with severe mental illness», Community Mental Health Journal, vol. 40, p. 75-90.

BERNSTEIN, M.A. et R. HENSLEY (1993). «Developing community-based program alternatives for serous and persistently mentally ill elderly», Journal of Mental Health Administration, vol. 20, 201-207.

BICKEL, J.-F. et S. CAVALLI (2002). «De I'exclusion dans les dernières étapes du parcours de vie. Un survol», Gérontologie et Société, vol. 102, p. 25-40.

BISHOP, J. et E. VINGILIS (2006). «Development of a framework for comprehensive evaluation of client outcomes in community mental health services», Canadian Journal of Program Evaluation, vol. 21, n², p. 133-180.

BLAIS, M. et S. Martineau (2006). «L'analyse inductive générale: description d'une démarche visant à donner un sens à des données brutes », Recherches qualitatives, vol. 26, n 2, p. 1-18.

CENTER ADDICTION MENTAL HEALTH (CAMH) (2009). «Best-practice guideline for mental health promotion programs: older adults $55+»$, <http://knowledges.camh.net>.

CALSYN, R.J., G.A MORSE, W.D. KLINKENBERG et M.R. LEMMING (2005). «Client outcomes and the working alliance in assertive community treatment programs», Journal of Case Management, vol. 5, p. 199-202.

CENTRE DE TOXICOMANIE ET DE SANTÉ MENTALE (CTSM) (2007). Établir des rapports: L'intégration dans les domaines de la toxicomanie et de la santé mentale. Rapport annuel, <www.camh.net/fr/Publications/Strategic_ Planning_Annual_Reports/Annual_Reports/2007>.

COHEN, A. (2001). The effectiveness of mental health services in primary care: the view from the developing world, Geneva, WHO. 
CONTANDRIOPOULOS, A.P., G. POUVOURVILLE, J.P. POULLIER et D. CONTANDRIOPOULOS (2000). «À la recherche d'une troisième voie: les systèmes de santé au XXI ${ }^{\mathrm{e}}$ siècle», dans M.P. Pomey, J.P. Poullier, B. Lejeune (dir.), Santé publique: États des lieux, enjeux et perspectives, Paris, France, Ellipses Éditions Marketing, p. 637-667.

CRABTREE, B. et W. MILLER (1999). Doing Qualitative Research, 2 Edition, Newbury Park, CA, Sage Publication.

DALLAIRE, B., D. MIRANDA, N. MOSCOVITZ et A. GUÉRETTE (2003). Les interventions communautaires auprès des personnes âgées aux prises avec des problèmes de santé mentale sévères et persistants, rapport soumis au Fonds québécois de recherche sur la société et la culture (FQRSC).

DUNN, G. et R. BENTALL (2007). «Modelling treatment-effect heterogeneity in randomized controlled trials of complex interventions (psychological treatments)», Statistics in Medicine, vol. 26, n² 26, p. 4719-4745.

HEALTH EDUCATION UNIT COMMUNICATION (2002). Évaluation des programmes de promotion de la santé, <http://www.thcu.ca/consultation.htm>.

JENSON, J. (2004). Canada's New Social Risks: Directions for a New Social Architecture, CPRN Research Report F 43, Ottawa, Canadian Policy Research Networks.

KILBOURNE, A.M., J.R. CORNELIUS, X. HAN, G.L. HAAS, I. SALLOUM, J. CONIGLIARO et H. PINCUS (2005). «General-medical conditions in older patients with serious mental illness», American Journal of Geriatric Psychiatry, vol. 13, p. 250-254.

KIRBY, M. J. L. et W. J. KEON (2004). Mental Health, Mental Illness and Addiction: Issues and options for Canada (Report 3), Interim report of The Standing Senate Committee On Social Affairs, Science and Technology.

KLUG, G., G. HERMANN, B. FUCHS-NIEDER, M. PANZER, A. HAIDERSTIPACEK et S. PRIEBE (2010). "Effectiveness of home treatment for elderly people with depression: randomised controlled trial», British Journal of Psychiatry, vol. 197, p. 463-467

MINISTÈRE DE LA FAMILLE ET DES AÎNÉS (2008). Préparons l'avenir avec nos aînés - Rapport de la consultation publique sur les conditions de vie des aînés, <http://www.mfa.gouv.qc.ca/fr/ministere/publications/Pages/liste.aspx>.

MINISTÈRE DE LA SANTÉ ET DES SERVICES SOCIAUX (MSSS) (2010). Mental Health Action Plan. publication.msss.gouv.qc.ca

MINISTÈRE DE LA SANTÉ ET DES SERVICES SOCIAUX (MSSS) (2005). Mental Health Action Plan 2005-2010. publications.msss.gouv.qc.ca

MIRANDA, D., N. MOSCOVITZ et L. D'ALTILIA (2003). «Le programme communautaire de santé mentale pour les personnes de 60 ans et plus au CLSC René-Cassin à Montréal», Bulletin Équilibre en Tête, vol. 17, n², p. 5-7.

MOSCOVITZ, N. (2000). A community 60 + Multidisciplinary Mental Health team, CLSC René-Cassin, Institute of Social Gerontology of Quebec, Présentation et documents, CSSS Cavendish, Côte-St-Luc.

NIKOLOVA, R., M. CARIGNAN, N. MOSCOVITZ et L. DEMERS (2004). «The psychogeriatric and risk behaviour assessment scale (PARBAS)». Archives of Gerontology and Geriatrics, vol. 39, p. 187-200. 
NOUR, K., M. HÉBERT, J.-P. LAVOIE, A. REGENSTREIF et N. MOSCOVITZ (2011). Évaluation des services spécialisés en santé mentale pour les personnes âgées de 60 ans et plus au CSSS Cavendish, rapport déposé aux Instituts de recherche en santé.

NOUR, K., D. MIRANDA, A. REGENSTREIF, B. DALLAIRE, N. MOSCOVITZ, M. HÉBERT et D. WHITEHEAD (2010). «Guidelines for Community-Based Team Interventions for Seniors with Severe and Persistant Mental Health Problems Living in the Community», Intervention, vol. 133, n² 2, p. 98-109.

PHILLIPSON, C. (2012). «Globalisation, economic recession and social exclusion : policy challenges and responses», dans T. SCHARF et N. KEATING (dir.), From Exclusion to Inclusion in Old Age. A Global Challenge, Bristol, The Policy Press, p. 17-32.

REPPER, J. et R. PERKINS (2006). Social Inclusion and Recovery: A Model for Mental Health Practice, UK, Bailliere Tindall.

ROMANOW, R. J. (2002). Building on Values: The Future of Health Care in Canada, Ottawa, Health Canada.

THOMAS, D.R. (2006). «A general inductive approach for analyzing qualitative evaluation data», American Journal of Evaluation », vol. 27, n² 2, p. 237-246.

\section{NOTES}

1 Les problèmes de santé mentale désignent des diagnostics psychiatriques (ex. dépression majeure, schizophrénie) associés à une détresse et un dysfonctionnement significatifs et d'autres risques élevés, dont le décès et la perte de liberté. Ils peuvent engendrer des problématiques sévères et récurrentes: comportements à risque, faibles niveaux de fonctionnement et d'autonomie, aggravation des symptômes psychiatriques et médicaux, etc. (Kilbourne et al., 2005; Nour et al., 2011; Nikolova, Carignan, Moscovitz et Demers, 2004). On distinguera donc dans le texte les troubles mentaux graves lorsque le problème de santé mentale est chronique et les troubles mentaux légers lorsque le problème est associé à des situations problématiques transitoires.

2 I-CLSC : Système d'information sur la clientèle et les services CLSC.

3 Les nouvelles demandes de services qui entrent par le guichet d'accès sont évaluées par des infirmières et des intervenants sociaux. Dans cette évaluation, ils peuvent communiquer avec les personnes qui ont fait une demande de services (ou pour qui est faite une demande) pour obtenir des renseignements additionnels. Une fois que les demandes sont traitées au guichet d'accès et discutées avec les responsables cliniques, elles sont classifiées selon leur urgence et le type de services requis puis sont référées vers les services de $1^{\mathrm{e}}, 2^{\mathrm{e}}$ ou $3^{\mathrm{e}}$ ligne.

4 Une impression diagnostique est donnée par une psychologue sur le diagnostic probable de santé mentale. Celui-ci sera par la suite confirmé par un psychogériatre. 